University of Nebraska - Lincoln

DigitalCommons@University of Nebraska - Lincoln

2010

\title{
Anisotropy of domain growth in epitaxial ferroelectric capacitors
}

D. Wu

North Carolina State University, Raleigh

I. Vrejoiu

Max Planck Institute of Microstructure Physics, Weinberg 2, D-06120 Halle/Saale, Germany

M. Alexe

Max Planck Institute of Microstructure Physics, Weinberg 2, D-06120 Halle/Saale, Germany, m.alexe@warwick.ac.uk

Alexei Gruverman

University of Nebraska-Lincoln, agruverman2@unl.edu

Follow this and additional works at: https://digitalcommons.unl.edu/physicsgruverman

Part of the Physics Commons

Wu, D.; Vrejoiu, I.; Alexe, M.; and Gruverman, Alexei, "Anisotropy of domain growth in epitaxial ferroelectric capacitors" (2010). Alexei Gruverman Publications. 54.

https://digitalcommons.unl.edu/physicsgruverman/54

This Article is brought to you for free and open access by the Research Papers in Physics and Astronomy at DigitalCommons@University of Nebraska - Lincoln. It has been accepted for inclusion in Alexei Gruverman Publications by an authorized administrator of DigitalCommons@University of Nebraska - Lincoln. 


\title{
Anisotropy of domain growth in epitaxial ferroelectric capacitors
}

\author{
D. Wu, ${ }^{1}$ I. Vrejoiu, ${ }^{2}$ M. Alexe, ${ }^{2}$ and A. Gruverman ${ }^{3, a)}$ \\ ${ }^{1}$ Department of Physics, North Carolina State University, Raleigh, North Carolina 27695, USA \\ ${ }^{2}$ Max Planck Institute of Microstructure Physics, Weinberg 2, D-06120 Halle/Saale, Germany \\ ${ }^{3}$ Department of Physics and Astronomy, University of Nebraska, Lincoln, Nebraska 68588-0111, USA
}

(Received 19 January 2010; accepted 28 February 2010; published online 17 March 2010)

\begin{abstract}
Piezoresponse force microscopy (PFM) has been applied to investigate the switching kinetics in microscale epitaxial $\mathrm{Pb}(\mathrm{Zr}, \mathrm{Ti}) \mathrm{O}_{3}$ capacitors. It is shown that transition from low to high field range brings about a qualitative change in domain growth kinetics, namely, laterally isotropic growth in the high fields as opposed to highly anisotropic growth in the low fields. It is suggested that anisotropy of domain growth can be attributed to orientational variations in the activation energy due to film microstructure. Fitting the switching kinetics using the Kolmogorov-Avrami-Ishibashi model shows excellent agreement with the PFM experimental data and yields the integer values of domain dimensionality. (C) 2010 American Institute of Physics. [doi:10.1063/1.3366724]
\end{abstract}

A driving force behind the development of integrated ferroelectric-semiconductor structures is the prospect of advanced devices with increased functionality, speed, endurance, and reliability. A physical basis of operation for most of the ferroelectric-based devices is the electrically induced polarization reversal which on a microscopic level occurs via the nucleation and growth of a number of domains with opposite polarization direction. In spite of continuous studies by means of integral electrical measurements, such as polarization hysteresis and transient current, there is still lack of information on the kinetics of domain nucleation and wall motion during polarization reversal, particularly in mesoscale and nanoscale structures with constrained geometries. In most ferroelectric-based devices, active elements are implemented in the form of capacitors where a thin layer of a ferroelectric material is sandwiched between two electrodes. Micrometer range sizes and switching times below $100 \mathrm{~ns}$ require testing of the switching processes in these capacitors at the appropriate spatial and time scales. ${ }^{1-4}$

Over the last several years, piezoresponse force microscopy (PFM) has become a commonly used technique for nanoscale studies of static and local switching properties of ferroelectric materials. ${ }^{5,6}$ Even the greater strength of PFM is its ability to detect the polarization state in ferroelectric capacitors by imaging ferroelectric domains through the top electrode ${ }^{7-10}$ A problem of low time resolution of PFM has been recently circumvented by using a stroboscopic PFM approach based on PFM visualization of domain configurations developing during a step-by-step polarization reversal. ${ }^{11-13}$ Direct imaging of domain kinetics provides a possibility to understand the discrepancy between switching behavior in epitaxial and polycrystalline ferroelectric films and to refine the existing models. A nucleation theory proposed by Kolmogorov and Avrami, and extended by Ishibashi ${ }^{14}$ - the KAI model—provides adequate description of the switching behavior in epitaxial thin films and single crystals. On the other hand, for polycrystalline thin film capacitors the nucleation-limited (NLS) model featuring an exponentially wide variation in nucleation times yields a much better fitting than the KAI model. ${ }^{15,16}$

${ }^{a)}$ Electronic mail: alexei_gruverman@unl.edu.
In this work, we report direct studies of the field-induced changes in domain switching kinetics by means of stroboscopic PFM. ${ }^{12}$ It is shown that in spite of the finite size of the capacitors used in this study, the statistical KAI model describes the experimentally measured switching behavior quite well. Fitting by the KAI model yields the parameters describing domain growth dimensionality close to integer values.

Domain switching kinetics have been studied in epitaxial $50 \mathrm{~nm}$ thick (001) $\mathrm{Pb}\left(\mathrm{Zr}_{0.2}, \mathrm{Ti}_{0.8}\right) 0_{3}$ (PZT) capacitors fabricated by pulsed laser deposition on $\mathrm{SrRuO}_{3} / \mathrm{SrTiO}_{3}$ (001) substrates. Details on film fabrication and basic properties are given elsewhere. ${ }^{17}$ Electron beam lithography and lift-off have been used to fabricate $50 \mathrm{~nm}$ thick Pt electrodes with $2.5 \times 2.5 \mu \mathrm{m}^{2}$ lateral dimensions on top of the PZT film.

The stroboscopic PFM approach is based on detection of the instantaneous domain patterns appearing in the ferroelectric capacitor after application of an input voltage pulse with an amplitude above the coercive voltage and duration shorter than the total switching time. ${ }^{12}$ The input pulse duration was varied in the range from $20 \mathrm{~ns}$ to several microseconds. At the beginning of each switching cycle, the capacitor was switched into the opposite polarization state by applying a set pulse $(-/+4 \mathrm{~V}, 1 \mathrm{~s})$. To counteract a training effect and slight imprint in the capacitors, a dc offset of $0.05 \mathrm{~V}$ was applied to the capacitors during PFM imaging.

It has been shown, that in each switching cycle, domain nucleation occurs in the predetermined sites most likely corresponding to the local defects at the film-electrode interface. This effect is a direct illustration of the fact that ferroelectric



FIG. 1. PFM images of instantaneous domain configurations developing at different stages of polarization reversal in the $2.5 \mu \mathrm{m}$ edge square capacitor under the electric field of $700 \mathrm{kV} / \mathrm{cm}$. 

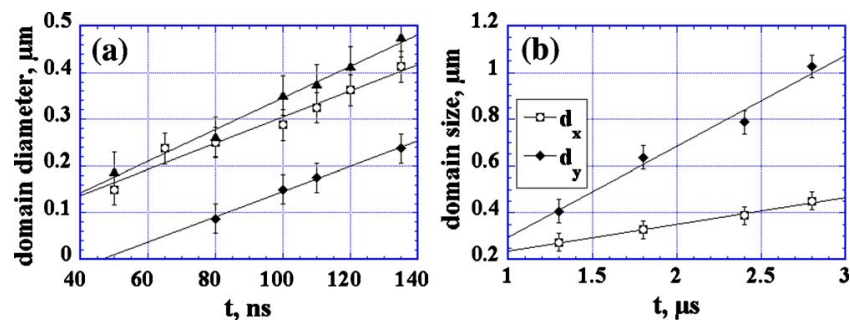

FIG. 2. (Color online) Time dependence of the growing domain size for an applied field of (a) $700 \mathrm{kV} / \mathrm{cm}$ (measured for three different domains in arbitrary directions); (b) $500 \mathrm{kV} / \mathrm{cm}$.

switching is a result of heterogeneous nucleation. Due to the reproducibility of domain switching kinetics, its characteristics such as nucleation rate and domain wall velocity can be calculated from the PFM snapshots taken at different time intervals.

Snapshots of domain patterns developing in PZT capacitors during switching under an applied field $E$ of $700 \mathrm{kV} / \mathrm{cm}$ are shown in Fig. 1. In this field range, switching occurs via nucleation of a number of domains followed by their lateral expansion. The switching occurs via dynamics of $180^{\circ}$ domains and no $90^{\circ}$ wall formation has been observed in agreement with earlier findings based on transient current measurements. ${ }^{18}$ The time-dependent evolution of domain structure reveals that the wall velocity is isotropic and independent of domain size in the range at least up to $400 \mathrm{~nm}$ in diameter [Fig. 2(a)].

Interestingly, significant anisotropy of the wall velocity has been detected during switching in the lower field range $(<500 \mathrm{kV} / \mathrm{cm})$. Measurements of the time dependencies of growing domain size [Fig. 2(b)] show that in the $y$-axis direction the wall velocity $v_{y}$ is almost three times higher than velocity $v_{x}$ in the $x$-axis direction $(0.39$ and $0.12 \mathrm{~m} / \mathrm{s}$, respectively). This anisotropy of wall velocity is manifested by the formation of characteristically elongated domains (Fig. 3). Overall, the switching kinetics in the low fields is characterized by a long-range (up to $1 \mu \mathrm{m}$ ) lateral growth of just a few nucleated domains in stark contrast to the switching in the high fields.

To quantify the switching kinetics, a time dependence of the switched capacitor volume fraction has been obtained via image analysis of the PFM data. ${ }^{12}$ Figure 4 shows timedependent plots of the switched capacitor volume for two different values of the applied field. In the KAI model, the volume fraction of the polarization switched by time $t$ is described by

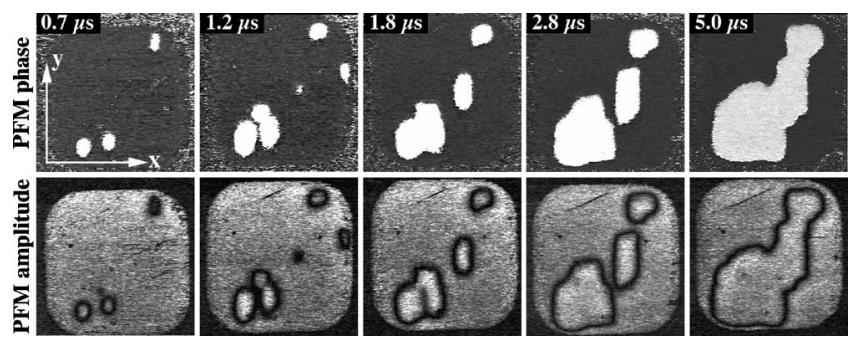

FIG. 3. PFM images of instantaneous domain configurations developing at different stages of polarization reversal in the $2.5 \mu \mathrm{m}$ edge square capacitor under the electric field of $500 \mathrm{kV} / \mathrm{cm}$.

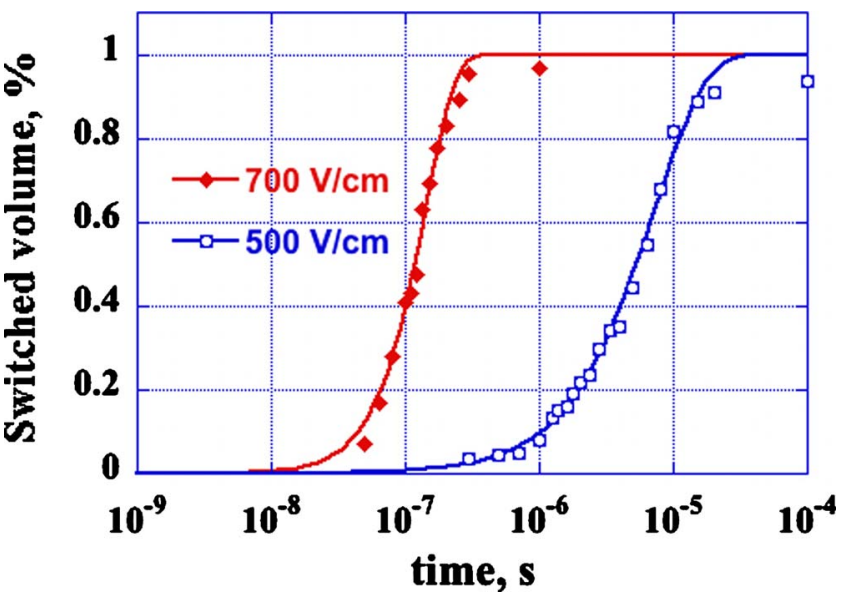

FIG. 4. (Color online) Fitting of the polarization switching kinetics by the KAI model. Domain dimensionality $n$ is 1.96 for $E=700 \mathrm{kV} / \mathrm{cm}$ and 1.12 for $E=500 \mathrm{kV} / \mathrm{cm}$.

$$
p(t)=1-\exp \left[-\left(t / t_{0}\right)^{n}\right],
$$

where $t_{0}$ is the characteristic switching time and $n$ can be treated as domain dimensionality reflecting the mechanism of polarization reversal. The solid lines in Fig. 4 show fitting of the PFM data by the KAI model. Remarkably, the KAI model provides excellent description of the switching behavior, in contrast to the case of polycrystalline PZT capacitors. ${ }^{12,19}$ It is found that $n$ values are different for low and high fields and are very close to integer values: 1.12 for $500 \mathrm{kV} / \mathrm{cm}$ and 1.96 for $700 \mathrm{kV} / \mathrm{cm}$, indicating a change in the switching mechanism. In the KAI model, $n=2$ corresponds to a growth of cylindrical domains (two-dimensional growth) and $n=1$ indicates a lateral expansion of lamellar domains (one-dimensional growth). An increase in $n$ with an applied field, also reported by So et al. ${ }^{20}$ is consistent with the change in domain kinetics revealed by stroboscopic PFM studies. Indeed, in the low fields, after the growing domains reach the capacitor edges perpendicular to the $y$-axis, they mostly grow in the $x$-axis direction, which can be considered as a transition from two-dimensional to one-dimensional domain growth.

To get a further insight into the domain switching, we performed local ferroelectric switching and imaging using an immobilized tip positioned outside of the top electrode on the bare PZT film surface. In Fig. 5(a), it can be seen that domains produced by a biased tip are of circular shape, i.e., exhibit isotropic growth behavior. Given that the field gener-

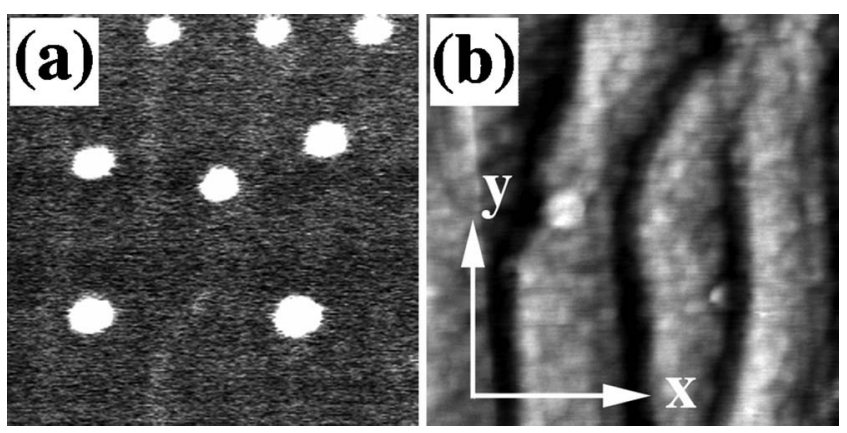

FIG. 5. (a) Domains generated by voltage pulses applied to the PFM tip fixed in various locations of the bare PZT surface. (b) Topographic image of the bare PZT film surface (outside of the top electrode). The scan size is $3 \times 3 \mu \mathrm{m}^{2}$. 
ated by the probing PFM tip at the tip-surface contact is of the order of several megavolts per centimeter, this result is consistent with the isotropic domain growth observed in the capacitors in the high field range. Interestingly, topographic imaging of the PZT film reveals characteristic morphological ridges with the ridge orientation coinciding with the direction of the fast domain growth in the low field range [Fig. 5(b)]. The most likely reason for this PZT film morphology is step bunching (with the steps reaching several unit cells in height) developed in the epitaxial $\mathrm{SrRuO}_{3}$ film grown on the vicinal $\mathrm{SrTiO}_{3}$ (100) surface. Consequently, the epitaxial PZT film grown on this step-bunched $\mathrm{SrRuO}_{3}$ underlayer followed its morphology. As the crystal symmetry of the tetragonal PZT film cannot be invoked to explain the domain growth anisotropy, one can assume that these morphological features are the main reason for the observed effect. Due to the smaller energy change associated with the nuclei formation at the morphological steps than at the terraces, domain wall motion controlled by nucleation of this sort occurs at a higher rate leading to faster domain growth along the steps.

The lateral domain wall motion can be considered a creep process, which is a generalization of the nucleation model by Miller and Weinreich: ${ }^{21}$

$$
v=v_{\infty} \exp \left(-\frac{\Delta U}{k_{B} T}\right)\left(\frac{E_{0}}{E}\right)^{\mu},
$$

where $\mu$ is a dynamic exponent and $\Delta U$ is an activation energy. Measurements of the transient currents as a function of the applied field revealed a typical exponential field dependence of the switching time with the activation field $E_{0}$ equal to $4.8 \mathrm{MV} / \mathrm{cm}$ and dynamic exponent $\mu=1$. From Eq. (2), the ratio of $v_{y} / v_{x}$ can be written as

$$
\frac{v_{y}}{v_{x}}=\exp \left(-\frac{\Delta U_{y}-\Delta U_{x}}{k T}\right),
$$

where $\Delta U_{y}$ and $\Delta U_{x}$ are the activation energies for nucleation at the domain walls perpendicular to axes $y$ and $x$, respectively. Since $\Delta U$ can be estimated to be about $10 \mathrm{kT}$, Eq. (3) then can be rewritten as

$$
\frac{\Delta U_{x}}{\Delta U_{y}}=1+0.1 \ln \left(\frac{v_{y}}{v_{x}}\right) .
$$

From Eq. (4) and experimental values of $v_{y}$ and $v_{x}$, one finds $\Delta U_{x} / \Delta U_{y}=1.12$. In other words, orientational variations in the activation energy by about $12 \%$ would cause anisotropy of domain wall velocity consistent with experimentally measured values. In the high field range, where $\Delta U$ becomes smaller, ${ }^{21}$ the difference between $\Delta U_{y}$ and $\Delta U_{x}$ decreases leading to the more isotropic growth of domains.

In summary, heterogeneous nature of domain nucleation during ferroelectric switching and reproducibility of the switching behavior allowed visualization of domain kinetics in epitaxial PZT capacitors with high space and time resolution by means of stroboscopic PFM. It is directly shown that transition from low to high fields results in qualitative change in domain switching kinetics: in the low field range domain growth is characterized by strong anisotropy of domain velocity while in the high field range domains exhibit isotropic two-dimensional growth behavior. Anisotropic domain growth can be attributed to the orientational dependence of activation energy due to the specific morphology of the PZT layer. The nucleation KAI model is in excellent agreement with the PFM switching data yielding parameters $n$ close to integer values. A change in the $n$ value with the field is consistent with the change in the domain growth dimensionality.

This work was supported by the National Science Foundation (Grant Nos. MRSEC DMR-0820521 and NIRT DMR0403871).

${ }^{1}$ J. F. Scott, Ferroelectric Memories (Springer, Berlin, 2000).

${ }^{2}$ J. Li, B. Nagaraj, H. Liang, W. Cao, C. H. Lee, and R. Ramesh, Appl. Phys. Lett. 84, 1174 (2004).

${ }^{3}$ S. Tiedke, T. Schmitz, K. Prume, A. Roelofs, T. Schneller, U. Kall, R. Waser, C. Ganpule, V. Nagarajan, A. Stanishefsky, and R. Ramesh, Appl. Phys. Lett. 79, 3678 (2001).

${ }^{4}$ A. Grigoriev, D.-H. Do, D. M. Kim, C.-B. Eom, B. Adams, E. Dufresne, and P. G. Evans, Phys. Rev. Lett. 96, 187601 (2006).

${ }^{5}$ Nanoscale Characterization of Ferroelectric Materials: Scanning Probe Microscopy Approach, edited by M. Alexe and A. Gruverman (Springer, Berlin, 2004).

${ }^{6}$ S. Jesse, A. P. Baddorf, and S. V. Kalinin, Appl. Phys. Lett. 88, 062908 (2006).

${ }^{7}$ A. Gruverman, B. J. Rodriguez, R. J. Nemanich, A. I. Kingon, J. S. Cross, and M. Tsukada, Appl. Phys. Lett. 82, 3071 (2003).

${ }^{8}$ S. V. Kalinin, B. J. Rodriguez, S.-H. Kim, S.-K. Hong, A. Gruverman, and E. A. Eliseev, Appl. Phys. Lett. 92, 152906 (2008).

${ }^{9}$ I. Stolichnov, E. Colla, A. Tagantsev, S. S. N. Bharadwaja, S. Hong, N. Setter, J. Cross, and M. Tsukada, Appl. Phys. Lett. 80, 4804 (2002).

${ }^{10}$ S. Hong, E. L. Colla, E. Kim, D. V. Taylor, A. K. Tagantsev, P. Muralt, K. No, and N. Setter, J. Appl. Phys. 86, 607 (1999).

${ }^{11}$ A. Gruverman, B. J. Rodriguez, C. Dehoff, J. D. Waldrep, A. I. Kingon, R. J. Nemanich, and J. S. Cross, Appl. Phys. Lett. 87, 082902 (2005).

${ }^{12}$ A. Gruverman, D. Wu, and J. F. Scott, Phys. Rev. Lett. 100, 097601 (2008).

${ }^{13}$ S. M. Yang, J. Y. Jo, D. J. Kim, H. Sung, T. W. Noh, H. N. Lee, J.-G. Yoon, and T. K. Song, Appl. Phys. Lett. 92, 252901 (2008).

${ }^{14}$ Y. Ishibashi and Y. Takagi, J. Phys. Soc. Jpn. 31, 506512 (1971).

${ }^{15}$ X. F. Du and I. W. Chen, Appl. Phys. Lett. 72, 1923 (1998).

${ }^{16}$ A. Tagantsev, I. Stolichnov, N. Setter, J. S. Cross, and M. Tsukada, Phys. Rev. B 66, 214109 (2002).

${ }^{17}$ I. Vrejoiu, G. Le Rhun, L. Pintilie, D. Hesse, M. Alexe, and U. Goesele, Adv. Mater. 18, 1657 (2006).

${ }^{18}$ W. Li and M. Alexe, Appl. Phys. Lett. 91, 262903 (2007).

${ }^{19}$ J. Y. Jo, H. S. Han, J.-G. Yoon, T. K. Song, S.-H. Kim, and T. W. Noh, Phys. Rev. Lett. 99, 267602 (2007).

${ }^{20}$ Y. W. So, D. J. Kim, T. W. Noh, J.-G. Yoon, and T. K. Song, Appl. Phys. Lett. 86, 092905 (2005).

${ }^{21}$ R. C. Miller and G. Weinreich, Phys. Rev. 117, 1460 (1960). 